\title{
Effects and safety profile of betahistine in patients in the Russian contingent of OSVaLD, an open- label observational study in vestibular vertigo
}

This article was published in the following Dove Press journal:

International Journal of General Medicine

22 January 2015

Number of times this article has been viewed

\section{Svetlana Vyacheslavovna \\ Morozova' \\ Natalia Stepanovna \\ Alekseeva $^{2}$ \\ Sergey Vasilyevich Lilenko ${ }^{3}$ \\ Eduard Ivanovich Matsnev ${ }^{4}$ \\ Oleg Anatol'evich Melnikov \\ 'Department of Ear, Nose, and Throat, State Budgetary Educational Institution of Higher Professional Training, IM Sechenov First Moscow State Medical University of the Ministry of Healthcare and Social Development of the Russian Federation, Moscow, ${ }^{2}$ Federal State Budgetary Institution, Scientific Neurology Center of the Russian Academy of Medical Sciences, Moscow, ${ }^{3}$ St Petersburg Research Institute of Ear, Throat, Nose and Speech, St Petersburg, ${ }^{4}$ Department of Physiology and Pathology of Auditory and Vestibular Systems, Federal Scientific Center (FSC), Institute for Biomedical Problems, Russian Academy of Sciences (RAS), Moscow, ${ }^{5}$ ANO Guta Clinic, Moscow, Russian Federation}

Correspondence: Sergey Vasilyevich Lilenko St Petersburg Research Institute of Ear, Throat, Nose and Speech, Bronnitskaya 9, St Petersburg, Russian Federation Email lilenko@mail.ru
Background: We report here data from the $>200$ patients recruited in Russia to take part in OSVaLD, a 12-week, open-label, post-marketing surveillance study of the response to betahistine $48 \mathrm{mg} /$ day in vertigo of peripheral vestibular origin carried out in a total of 13 countries.

Methods: The primary efficacy endpoint was change in the Dizziness Handicap Inventory (DHI; 100-point scale). Changes in Hospital Anxiety and Depression Scale (HADS) and Medical Outcomes Study Short-Form 36, version 2 (SF-36v2 ${ }^{\circledR}$ ) scores were a priori secondary Outcomes. Results: Total DHI score improved by 43 points during betahistine treatment. This aggregate improvement was equally distributed across the three domains of the DHI (physical, emotional, and functional; $P<0.0001$ for main and subscore changes from baseline). Statistically significant improvements versus baseline were also observed in mean HADS scores for anxiety and depression (both $P<0.0001$ ), and in the Physical Component Summary and Mental Component Summary scores of the SF-36v2 (both $P<0.0001$ versus baseline). Only one suspected adverse drug reaction was recorded in the Russian safety population $(n=204)$, indicating that betahistine was well tolerated in those patients.

Conclusion: Betahistine $48 \mathrm{mg} /$ day was associated with clear improvements in well-configured and widely validated measures of health-related quality of life and an encouraging tolerability profile in patients in Russia who took part in OSVaLD.

Keywords: betahistine, vertigo, dizziness, anxiety, depression, quality of life, Russia

\section{Introduction}

Improvements in health-related quality of life (HRQoL) in patients of 13 countries who were prescribed betahistine for recurrent peripheral vestibular vertigo have been reported from the OSVaLD study (a three-month Observational Study in patients suffering from recurrent peripheral vestibular Vertigo to assess the effect of betahistine $48 \mathrm{mg} /$ day on quality of Life and Dizziness symptoms). ${ }^{1,2}$

One of the largest single-country contingents of OSVaLD was recruited in Russia, and there is interest among physicians in Russia in having detailed information about their national contingent. In response to that interest, we report here the findings from the Russian participants in OSVaLD.

\section{Materials and methods}

The methods used in OSVaLD have been described in detail in other papers. ${ }^{1,2}$ Readers should consult those sources for information about the statistical principles that shaped the study and the statistical methods used to analyze the data, including prospectively defined arrangements for dealing with missing data. Summarized in brief, OSVaLD was 
a post-marketing surveillance study of open-label betahistine; the study was scheduled to last 12 weeks. Participating patients were recruited at multiple primary care centers in 13 countries, including Russia.

Inclusion criteria were simple, comprising a history ( $\leq 5$ years) of vertigo attacks of peripheral vestibular origin and a baseline total score $\geq 40$ on the Dizziness Handicap Inventory (DHI). The only exclusion condition specified in the study protocol was if a patient satisfied one or more of the officially acknowledged contraindications to the use of betahistine.

Participating physicians were instructed to prescribe betahistine at a dose of $48 \mathrm{mg} /$ day; this was to be given in two or three equal divided doses according to local regulations and practice. Betahistine could be prescribed as sole therapy or added to existing antivertigo medications. Individual investigators were free to decide whether to continue or discontinue drugs that were being prescribed before the start of the study.

Patients were to attend three clinic visits for assessment of their response to betahistine: a baseline visit, and two follow-up visits at months 1 and 3. If a patient discontinued the trial before any scheduled visit, an end-of-treatment visit was arranged.

Endpoints included HRQoL, quantified using scores on the DHI, the Medical Outcomes Study Short-Form 36, version $2\left(\mathrm{SF}-36 \mathrm{v} 2^{\circledR}\right)$ and the Hospital Anxiety and Depression Scale (HADS); these data were recorded at each visit. The primary efficacy outcomes defined in the protocol was the change in total DHI score between baseline and 12 weeks (or end of study if earlier). All these instruments are widely used and documented for measuring HRQoL. ${ }^{3-12}$ Reports of adverse drug reactions were accrued from the safety population.

\section{Data collation and analysis}

Data management and statistical analysis was undertaken by the FOVEA Group (Rueil Malmaison, France). Microsoft Access version 9.0 was used for data entry, and SAS version 8.2 was used for quality control and statistical analysis. SF Health Outcomes ${ }^{\mathrm{TM}}$ scoring software (QualityMetric Incorporated, Lincoln, RI, USA) was used for some statistical analyses.

\section{Ethics and patient consent}

The design and conduct of OSVaLD conformed to international principles of Good Clinical Practice and the Declaration of Helsinki, and included independent institutional review of the protocol and securing advance informed consent from all patients where required by local laws. Pre-enrolment advice to patients included informing them that they could leave the study at any time without giving a reason and with no detriment to their care.

\section{Results}

In Russia, a total of 204 patients were recruited at 34 centers (see Acknowledgments section for details of participating practitioners). All 204 patients were included in both the efficacy population (patients who were prescribed betahistine at baseline, attended at least one clinic visit after baseline, and recorded at least one score for at least one endpoint at baseline and during at least one later visit) and the safety population (all patients who were prescribed betahistine at baseline and attended at least one later clinic visit). Table 1 summarizes the demographic profile of the Russian cohort, which was almost entirely white/Caucasian (98\%) and predominantly female.

Insufficient efficacy of existing therapy was recorded as the reason for prescribing betahistine in 119 cases $(58.3 \%)$ in the Russian contingent; new diagnosis ( $n=85,41.7 \%)$ accounted for the remainder. No patient had betahistine prescribed for multiple reasons.

Betahistine was mostly prescribed in a $16 \mathrm{mg}$ three times daily regimen throughout the study ( $85 \%-90 \%$ of patients). The mean treatment duration was $91 \pm 5$ days.

Table I Demographic features of the Russian efficacy/safety populations in the OSVaLD study

\begin{tabular}{ll}
\hline Characteristic & $\begin{array}{l}\text { Efficacy/safety population } \\
(\mathbf{n}=\mathbf{2 0 4})\end{array}$ \\
\hline Sex, $\mathrm{n}(\%)$ & $55(27.0)$ \\
Male & $149(73.0)$ \\
Female & $50.8 \pm 13.3$ \\
Age (years), (mean \pm SD) & \\
Age group (years), $\mathrm{n}(\%)$ & $13(6.4)$ \\
I8-29 & $24(11.9)$ \\
$30-39$ & $57(28.2)$ \\
$40-49$ & $54(26.7)$ \\
$50-59$ & $41(20.3)$ \\
$60-69$ & $12(5.9)$ \\
$70-79$ & $1(0.5)$ \\
$80-89$ & $167 \pm 8.0$ \\
Height (cm), (mean \pm SD) & $74.4 \pm 14.0$ \\
Weight (kg), (mean \pm SD) & $26.5 \pm 4.3$ \\
BMI (kg/m²), (mean \pm SD) & \\
Qualifying diagnosis, $n(\%)$ & $139(68.1)$ \\
PVVUP & $36(17.6)$ \\
BPPV & $7(3.4)$ \\
Ménière's disease & $13(6.4)$ \\
Multiple diagnoses & $9(4.5)$ \\
Other &
\end{tabular}

Abbreviations: BMI, body mass index; PVVUP, peripheral vestibular vertigo of unknown pathophysiology; BPPV, benign paroxysmal positional vertigo; SD, standard deviation. 
This subset of OSVaLD patients had extensive comorbidity, with pre-existing cerebrovascular or cardiovascular diseases identified in $>50 \%$ and $>25 \%$ of patients, respectively, and metabolic disturbances (including diabetes) and psychosomatic/psychiatric disorders (including panic disorder) present in $5 \%-7 \%$ of patients. However, no patient was recorded as having a history of drug or alcohol abuse.

Among the single-diagnosis categories, combination therapy was noted in a higher percentage of patients with Ménière's disease $(\approx 57 \%)$ than those with peripheral vestibular vertigo of unknown pathophysiology (PVVUP) or benign paroxysmal positional vertigo (BPPV; both $\approx 30 \%$ ). Piracetam was prescribed for between $40 \%$ and $50 \%$ of patients with PVVUP (48.9\%) or BPPV (41.7\%), but was not used in the small number of patients with a sole diagnosis of Ménière's disease $(n=7)$; in contrast, gingko biloba was prescribed for approximately $25 \%$ of patients with PVVUP or Ménière's disease but in only one patient with a sole diagnosis of BPPV.

\section{Efficacy outcomes \\ $\mathrm{DHI}$}

Net mean changes (improvements) in total DHI score and all its dimensions are depicted in Figure $1(P<0.0001$ for all comparisons versus baseline). The DHI response was consistent in men and women, and across the diagnostic categories of PVVUP, BPPV, and Ménière's disease (data not shown).

The mean change in total DHI score was numerically smaller in patients prescribed betahistine alone $(n=127)$ than in those prescribed combination therapy

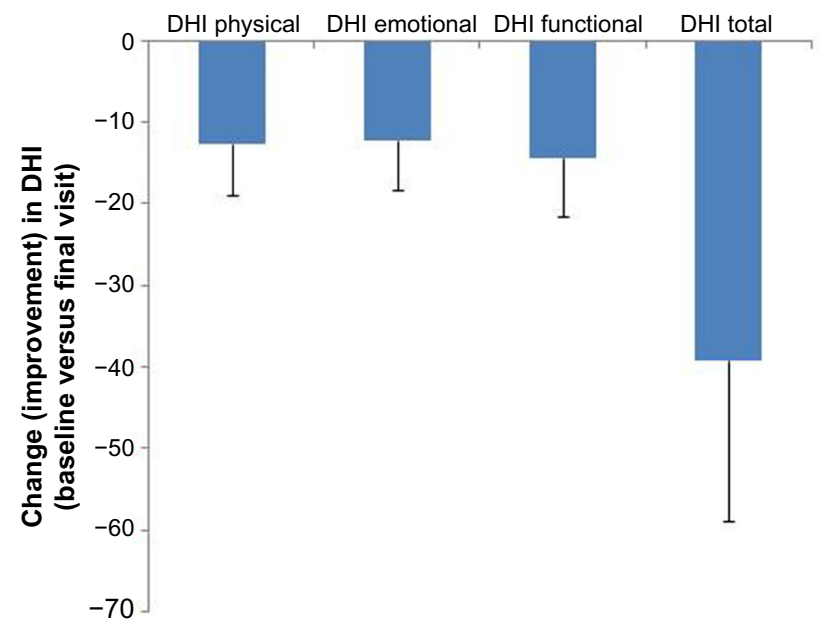

Figure I Changes from baseline in components of the $\mathrm{DHI}$ and in total $\mathrm{DHI}$ score in the Russian efficacy population of the OSVaLD study. Negative change values signify a reduced level of disability. $P<0.000$ I for all intradomain comparisons. Abbreviation: DHI, Dizziness Handicap Inventory.
( $\mathrm{n}=77 ; 36.5 \pm 18.3$ versus $43.6 \pm 15.3$ ), but comparisons versus baseline for the total DHI scores and for dimension-specific DHI scores were highly statistically significant $(P<0.0001)$ and indicative of improved HRQoL.

\section{SF-36v2}

Significant improvements from baseline were noted in the mean scores for both the Physical Component Summary (PCS) and the Mental Component Summary (MCS) subscales of the SF-36v2 (Table 2). Also as shown in Table 2, PCS and MCS scores improved to a similar extent in all major diagnostic categories. Similarly, there were improvements in all domains of the SF-36v2 (Figure 2, $P<0.0001$ ). Numerical differences were noted in the responses of men and women in some domains but these were small; statistical comparisons were not performed.

\section{HADS}

Trends in HADS scores are summarized in Table 3. Improvements were observed regardless of sex or diagnostic category.

\section{Subjective assessment of efficacy}

Patients with PVVUP were more likely than those with BPPV or Ménière's disease to assess treatment as "excellent" (43.2\% versus $33.3 \%$ and $28.6 \%$, respectively) but almost all patients in every category assessed betahistine therapy as "excellent" or "good" (Figure 3). Similarly, no meaningful differences in treatment ratings were seen between men and women. There was a good correlation between patients' impression of the treatment and the assessments of physicians $(r=0.725, P<0.0001)$.

Table 2 On-study trends in the PCS and MCS subscales of the SF- $36 \mathrm{v} 2^{\circledR}$ in the Russian efficacy population of the OSVaLD study

\begin{tabular}{lccc}
\hline SF-36v2 subscale & Baseline & $\begin{array}{l}\text { End of } \\
\text { study }\end{array}$ & $\begin{array}{l}\text { Change } \\
\text { (final - baseline) }\end{array}$ \\
\hline PCS & $41.8 \pm 7.1$ & $50.4 \pm 7$ & $8.7 \pm 7.4$ \\
PVVUP & $42 \pm 6.5$ & $50.3 \pm 6$ & $8.3 \pm 6.6$ \\
BPPV & $40.5 \pm 8.2$ & $51.2 \pm 8.5$ & $10.7 \pm 10.5$ \\
Ménière's disease & $44.5 \pm 5$ & $53.9 \pm 4.4$ & $9.4 \pm 3.7$ \\
MCS & $33.1 \pm 11$ & $48.3 \pm 8.7$ & $15.2 \pm 12.1$ \\
PVVUP & $32.3 \pm 10.4$ & $48.1 \pm 8.5$ & $15.8 \pm 12$ \\
BPPV & $33.6 \pm 11$ & $48.1 \pm 8.7$ & $14.5 \pm 13$ \\
Ménière's disease & $41 \pm 13$ & $52.1 \pm 8.7$ & $11.1 \pm 9.4$ \\
\hline
\end{tabular}

Notes: Data shown are mean \pm SD. Higher numbers signify better level of functioning. $P<0.000 \mathrm{I}$ for all comparisons of final versus baseline mean values.

Abbreviations: PVVUP, peripheral vestibular vertigo of unknown pathophysiology; BPPV, benign paroxysmal positional vertigo; SF-36v2, Medical Outcomes Study ShortForm 36, version 2; PCS, Physical Component Summary; MCS, Mental Component Summary; SD, standard deviation. 


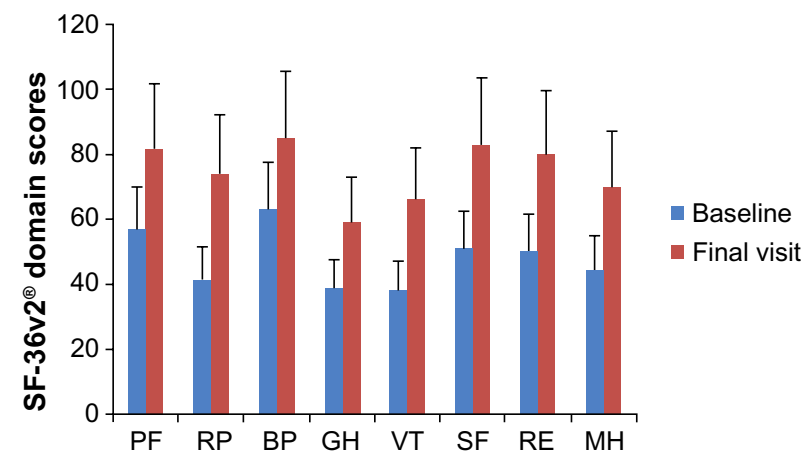

Figure 2 Changes from baseline in the domains of the SF-36v2 instrument in the Russian efficacy population of the OSVaLD study. Data shown are mean \pm SD. Higher numbers signify a better level of functioning. $P<0.000 \mathrm{I}$ for all intradomain comparisons.

Abbreviations: SF-36v2, Medical Outcomes Study Short-Form 36, version 2; PF, physical functioning; RP, role limitation physical; BP, bodily pain; $\mathrm{GH}$, general health perception; VT, vitality; SF, social functioning; RE, role limitation emotional; $\mathrm{MH}$, mental health; SD, standard deviation.

\section{Safety and tolerability}

The only suspected adverse drug reaction recorded in the Russian contingent was a report of nausea in a 37-year-old female patient. This event was recorded as possibly related to use of the study medication but was not classified as either severe or serious. In the study as a whole, more patients experienced a suspected adverse drug reaction at a betahistine dose of $24 \mathrm{mg}$ twice daily than at a dose of $16 \mathrm{mg}$ three times daily ( 25 versus 7; dosage data not recorded for 28 suspected adverse drug reactions). No deaths were reported during the study.

\section{Body weight}

Mean \pm standard deviation change in weight between the baseline and final visits in the efficacy population was $-0.4 \pm 3.2 \mathrm{~kg}$; change in body weight was almost identical in men and women. Patients with PVVUP ( $n=139$;

Table 3 Trends in HADS-A and HADS-D scores and distributions in the Russian efficacy population of the OSVaLD study $(n=204)$

\begin{tabular}{llll}
\hline HADS score & Baseline & $\begin{array}{l}\text { End of } \\
\text { study }\end{array}$ & $\begin{array}{l}\text { Change } \\
\text { (final - baseline) }\end{array}$ \\
\hline $\begin{array}{l}\text { HADS-Anxiety score } \\
\text { HADS-Anxiety level (\%) }\end{array}$ & $9.1 \pm 4$ & $4.8 \pm 3.6$ & $-4.3 \pm 4.3$ \\
$\quad$ Moderate & 30.4 & 5.4 & \\
$\quad$ Severe & 8.8 & 1.0 & \\
HADS-Depression score & $8 \pm 4.3$ & $3.7 \pm 3.6$ & $-4.3 \pm 4.8$ \\
HADS-Depression level (\%) & & & \\
$\quad$ Moderate & 20.1 & 5.9 & \\
$\quad$ Severe & 8.3 & 0.5 & \\
\hline
\end{tabular}

Notes: Data shown are mean \pm SD unless stated otherwise. Negative change values signify reduced level of disability. $P<0.000$ I for all comparisons of final versus baseline mean values.

Abbreviations: HADS-A, Hospital Anxiety and Depression Scale-Anxiety subscale; HADS-D, Hospital Anxiety and Depression Scale-Depression subscale; SD, standard deviation.

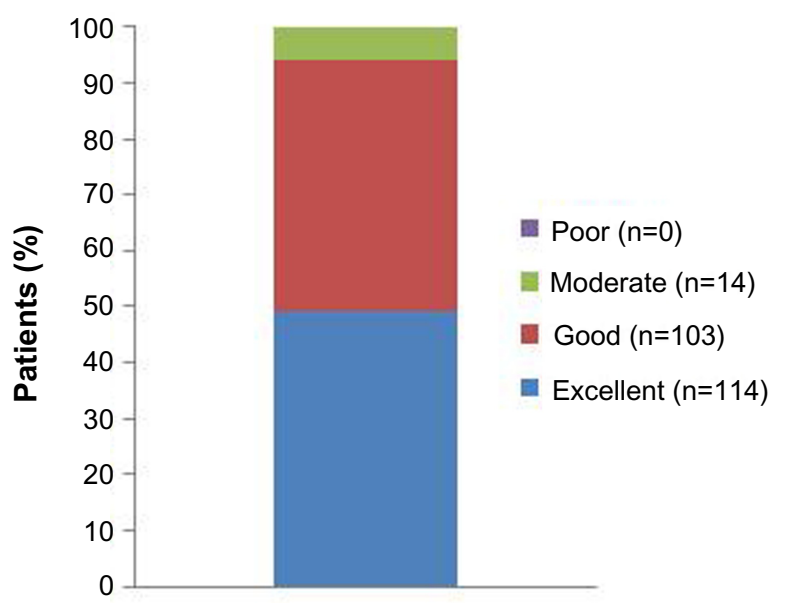

Figure 3 Patients' impression of betahistine therapy in the Russian efficacy population of OSVaLD.

$-0.5 \pm 3.4 \mathrm{~kg}$ ) lost more body weight, on average, than those with BPPV $(\mathrm{n}=36 ;-0.2 \pm 3.3 \mathrm{~kg})$ or Ménière's disease $(\mathrm{n}=7 ;-0.3 \pm 0.5 \mathrm{~kg})$.

\section{Discussion}

This exploratory analysis of the Russian contingent of the OSVaLD population replicates the demonstration of improved HRQoL with betahistine reported in the overall study. ${ }^{1}$ HRQoL data collected with three separate instruments in our Russian contingent of OSVaLD corroborate the premise that diseases of the peripheral vestibular system can have significant adverse impact on the lives of patients. ${ }^{13}$ Conversely, the improvements in all three indices recorded during the study indicate the possibility of intervening effectively to improve subjective perceptions of health and HRQoL.

Substantial improvements were seen in the primary efficacy criterion (absolute change from baseline in mean total DHI score between the baseline and final [3-month] visits), with a 39-point reduction in the mean total score, and mean reductions of 12.6, 12.2, and 14.4 points, respectively, in the physical, emotional, and functional domains of the DHI. All these improvements were statistically robust $(P<0.0001)$ and consistent across subgroups. Further, the improvement in total DHI score substantially exceeded the threshold for a minimally important change. ${ }^{14,15}$

Statistically significant $(P<0.0001)$ and clinically meaningful improvements were also recorded with the HADS questionnaire and the SF-36v2. This consistency of effect across scales gives us confidence that the responses seen are authentic, although the exploratory nature of the analysis must be borne in 
mind. It should be considered also that this Russian subgroup is characterized by patients with a diagnosis of PVVUP and that any conclusions drawn about the efficacy of betahistine are likely to be most resilient for that diagnosis.

Nevertheless, our findings are consistent with evidence from a meta-analysis of the beneficial effects of betahistine in Ménière's disease and vestibular vertigo ${ }^{16}$ and with local reports on the use of betahistine. ${ }^{17-19}$ Successful use of betahistine in the management of vertigo after a stroke has also been documented by Russian investigators, ${ }^{20}$ making it perhaps noteworthy that about half of the Russian OSVaLD contingent had a history of cerebrovascular disease.

Just over $40 \%$ of our patients were classified as having newly diagnosed vertigo. This may be pertinent to the analysis of our findings because it has been suggested that betahistine is more effective in BPPV when given to patients in whom the condition has not been apparent for long. ${ }^{21} \mathrm{We}$ have been unable to investigate this possibility in the Russian contingent of OSVaLD and would identify this as a limitation of our research.

Also untested in OSVaLD was the impact of higher doses of betahistine, especially in Ménière's disease. It is widely considered that a betahistine dose of $48 \mathrm{mg}$ three times daily should be used in individuals with this condition, ${ }^{22}$ which is three times higher than the dose examined in OSVaLD (48 $\mathrm{mg} /$ day); doses of up to $480 \mathrm{mg} /$ day have reportedly been used successfully in severe cases. ${ }^{23}$ It is possible, therefore, that the beneficial effects of betahistine on HRQoL in our database are not a full representation of the effects that might be achieved.

Connected with this is the question of whether long-term use of betahistine is appropriate and beneficial. For Ménière's disease, the answer appears to be yes, ${ }^{24,25}$ but in BPPV the principal benefit of the drug may be to provide accelerated symptom relief during the first months of treatment. ${ }^{26} \mathrm{We}$ consider it likely that the 3-month duration of OSVaLD was sufficient to explore and reveal much of the likely achievable change in HRQoL, and we consider that most or all of the changes seen may reasonably be attributed to betahistine use. However, reported rates of spontaneous complete resolution of BPPV can be high ${ }^{27}$ and, with small numbers and in the absence of a control group, the changes in HRQoL in the Russian contingent of OSVaLD are associated with betahistine use, not firm proof of cause and effect. Consideration must also be given to the absence of formal methods for monitoring patient adherence to prescribed therapies.

The open-label observational model of clinical research has limitations, ${ }^{28}$ notably the absence of a reference group, but it provided a pragmatic and reasonably robust methodology for a multinational trial performed in the setting of routine care and was compliant with the general principles of the Strengthening the Reporting of Observational Studies in Epidemiology methodology. ${ }^{29}$

Overall, the safety experience with betahistine in OSVaLD was satisfactory, with adverse drug reactions affecting $<2.5 \%$ of the study population. ${ }^{1}$ The predominant safety findings were gastrointestinal and nervous system disorders, as has been reported in earlier studies of betahistine; ${ }^{30}$ it has been reported that betahistine exhibits good tolerability in Ménière's disease even at ten times the dose used in our patients. ${ }^{23}$

There was notable national variation in the reporting of the rates of suspected adverse drug reactions in OSVaLD, with only one serious adverse drug reaction being recorded in the Russian contingent compared with 20 patients in Brazil. Some weight gain is a usual experience with betahistine, but in our Russian patients there was a small reduction in average weight. We are unable to say if this is a chance finding or a reflection of specific but unidentified local factors.

\section{Conclusion}

In 204 Russian patients diagnosed with recurrent peripheral vestibular vertigo, betahistine $48 \mathrm{mg}$ /day for 3 months was associated with sustained and statistically significant improvements in multiple indices of HRQoL. The safety and tolerability of the treatment were good in this cohort, with only one reported serious adverse drug reaction.

\section{Acknowledgments}

The authors wish to thank the physicians and patients who participated in the OSVaLD survey. A full listing of the investigators from 34 centers in Russia contributing data to this report appears here: Alekseeva N (Moscow), Artemova I (Moscow), Batysheva T (Moscow), Bobyreva S (Moscow), Boyko A (Moscow), Buldakova N (Moscow), Ganzhula P (Moscow), Gaponova O (Moscow), Hanevich T (Moscow), Hozova A (Moscow), Isachenkova O (Moscow), Ismailov A (Moscow), Zhuravleva E (Moscow), Kostenko E (Moscow), Lilenko S (St Petersburg), Lisenker L (Moscow), Makarova G (Moscow), Manevich T (Moscow), Matsnev E (Moscow), Melnikov O (Moscow), Morozova S (Moscow), Nesterova O (Moscow), Nikulina I (Moscow), Otcheskaya O (Moscow), Pivovarova V (Moscow), Rotor L (Moscow), Rylskiy A (Moscow), Shalabanova I (Moscow), Shinkarev S (Moscow), Sorokoumov V (St Petersburg), Vdovichenko $\mathrm{T}$ (Moscow), Vinetskiy Y (Moscow), Vostricova I (Moscow), Zadorozhnaya T (Moscow). Preparation of this report was assisted by Hughes associates, Oxford, UK. 


\section{Author contributions}

All the named authors made substantial contributions to the acquisition of data, and to its analysis and interpretation. They also contributed to drafting the article and/or revising it critically for important intellectual content. All named authors provided final approval of the version to be published and were accountable for ensuring that questions related to the accuracy or integrity of any part of the work were appropriately investigated and resolved.

\section{Disclosure}

OSVaLD is supported financially by Abbott Products Operations AG, Allschwil, Switzerland. The authors report no conflicts of interest in this work.

\section{References}

1. Benecke H, Pérez-Garrigues H, Bin Sidek D, et al; OSVaLD Investigators. Effects of betahistine on patient-reported outcomes in routine practice in patients with vestibular vertigo and appraisal of tolerability: experience in the OSVaLD study. Int Tinnitus J. 2010;16:14-24.

2. Pérez-Garrigues H, Kuessner D, Benecke H. Patient baseline characteristics in a multinational study of betahistine in recurrent peripheral vestibular vertigo: the OSVaLD study. Curr Med Res Opin. 2007;23:2753-2761.

3. Jacobson GP, Newman CW. The development of the Dizziness Handicap Inventory. Arch Otolaryngol Head Neck Surg. 1990;116:424-427.

4. Enloe LJ, Shields RK. Evaluation of health-related quality of life in individuals with vestibular disease using disease-specific and general outcomes measures. Phys Ther. 1997;77:890-903.

5. Jarlsäter S, Mattson E. Test of reliability of the dizziness handicap inventory and the activities-specific balance confidence scale for use in Sweden. Adv Physiother. 2003;5:137-144.

6. Kammerlind AS, Ledin TE, Skargren EI, Odkvist LM. Reliability of clinical balance tests and subjective ratings in dizziness and disequilibrium. Adv Physiother. 2005;7:96-107.

7. Ware JE Jr, Sherbourne CD. The MOS 36-item short-form health survey (SF-36): I. Conceptual framework and item selection. Med Care. 1992;30:473-483.

8. McHorney CA, Ware JE Jr, Raczek AE. The MOS 36-Item Short-Form Health Survey (SF-36): II. Psychometric and clinical tests of validity in measuring physical and mental health constructs. Med Care. 1993;31:247-263.

9. McHorney CA, Ware JE Jr, Lu JF, Sherbourne CD. The MOS 36-item Short-Form Health Survey (SF-36): III. Tests of data quality, scaling assumptions, and reliability across diverse patient groups. Med Care. 1994;32:40-66.

10. Saris-Baglama RN, Dewey CJ, Chisholm GB, et al. SF Health Outcomes $^{\mathrm{TM}}$ Scoring Software User's Guide. Lincoln, RI, USA: QualityMetric Inc; 2004.

11. SF-36v2 ${ }^{\mathrm{TM}}$. Scoring SF-36 Scales. Lincoln, RI, USA: QualityMetric Inc.; 2000.

12. Zigmond AS, Snaith RP. The Hospital Anxiety and Depression Scale. Acta Psychiatr Scand. 1983;67:361-370.
13. Benecke H, Agus S, Kuessner D, Goodall G, Strupp M. The burden and impact of vertigo: findings from the REVERT Patient Registry. Front Neurol. 2013;4:136.

14. Whitney SL, Hudak MT, Marchetti GF. The activities-specific balance confidence scale and the dizziness handicap inventory: a comparison. J Vestib Res. 1999;9:253-259.

15. Tamber AL, Wilhelmsen KT, Strand LI. Measurement properties of the Dizziness Handicap Inventory by cross-sectional and longitudinal designs. Health Qual Life Outcomes. 2009;7:101.

16. Nauta JJ. Meta-analysis of clinical studies with betahistine in Ménière's disease and vestibular vertigo. Eur Arch Otorhinolaryngol. 2014;271:887-897.

17. Kostenko EV, Petrova LV, Torgovanova EA, et al. [Treatment of vestibular vertigo and Ménière syndrome in outpatient clinics]. Zh Nevrol Psikhiatr Im S S Korsakova. 2012;112:36-40. Russian.

18. Gornostaeva GV, Varakin IuIa, Prokopovich ME, Alekseeva NS, Fedin PA, Chechetkin AO. [Epidemiology, clinical features and Betaserc therapy of vertigo in initial and reversible cerebrovascular pathology]. Zh Nevrol Psikhiatr Im S S Korsakova. 2005;105:14-17. Russian.

19. Larikova TI, Cherevikova GM. [Betaserc and improvement of life quality in war veterans]. Zh Nevrol Psikhiatr Im S S Korsakova. 2005;105:68-70. Russian.

20. Gekht AB, Vialkova AB, Galanov DV. [Clinico-neurological and stabilometric analysis of betahistine (Betaserc) efficacy in patients with vertigo in the rehabilitation period of ischemic stroke]. Zh Nevrol Psikhiatr Im S S Korsakova. 2005; Suppl 15:32-38. Russian.

21. Stambolieva K, Angov G. Effect of treatment with betahistine dihydrochloride on the postural stability in patients with different duration of benign paroxysmal positional vertigo. Int Tinnitus $J$. 2010;16:32-36.

22. Strupp M, Kremmyda O, Brandt T. Pharmacotherapy of vestibular disorders and nystagmus. Semin Neurol. 2013;33:286-296.

23. Lezius F, Adrion C, Mansmann U, Jahn K, Strupp M. High-dosage betahistine dihydrochloride between 288 and $480 \mathrm{mg}$ /day in patients with severe Ménière's disease: a case series. Eur Arch Otorhinolaryngol. 2011;268:1237-1240.

24. Strupp M, Hupert D, Frenzel C, et al. Long-term prophylactic treatment of attacks of vertigo in Ménière's disease - comparison of a high with a low dosage of betahistine in an open trial. Acta Otolaryngol. 2008; 128:520-524.

25. Strupp M, Brandt T. Diagnosis and treatment of vertigo and dizziness. Dtsch Arztebl Int. 2008;105:173-180.

26. Cavalieri M, Mottola G, Iemma M. Benign paroxysmal positional vertigo: a study of two manoeuvres with and without betahistine. Acta Otorhinolaryngol Ital. 2005;25:107-112.

27. Hornibrook J. Benign paroxysmal positional vertigo (BPPV): history, pathophysiology, office treatment and future directions. Int $J$ Otolaryngol. 2011;2011:835671.

28. Gauthier S, Juby A, Morelli L, Rehel B, Schecter R. A large, naturalistic, community-based study of rivastigmine in mild-to-moderate $\mathrm{AD}$ : the EXTEND Study. Curr Med Res Opin. 2006;22:2251-2265.

29. von Elm E, Altman DG, Egger M, Pocock SJ, Gøtzsche PC, Vandenbroucke JP. The Strengthening the Reporting of Observational Studies in Epidemiology (STROBE) statement: guidelines for reporting observational studies. PLoS One. 2007;4:e296.

30. Jeck-Thole $S$, Wagner $W$. Betahistine: a retrospective synopsis of safety data. Drug Saf. 2006;29:1049-1059. 
International Journal of General Medicine

Dovepress

\section{Publish your work in this journal}

The International Journal of General Medicine is an international, peer-reviewed open-access journal that focuses on general and internal medicine, pathogenesis, epidemiology, diagnosis, monitoring and treatment protocols. The journal is characterized by the rapid reporting of reviews, original research and clinical studies across all disease areas.
A key focus is the elucidation of disease processes and management protocols resulting in improved outcomes for the patient. The manuscript management system is completely online and includes a very quick and fair peer-review system. Visit http://www.dovepress.com/ testimonials.php to read real quotes from published authors.

Submit your manuscript here: http://www.dovepress.com/international-journal-of-general-medicine-journal 